\title{
Per pochi e in condizioni specifiche: L'esperienza del bilancio partecipativo (BP) nel comune di Milano
}

\author{
Cellini Marco ${ }^{\mathrm{a} *}$ and M. C. Antonucci ${ }^{\mathrm{b}}$
}

Istituto di Ricerche sulla Popolazione e le Politiche Sociali, Consiglio Nazionale delle Richerche (IRPPS-CNR), Roma, Italia; Istituto di Ricerche sulla Popolazione e le Politiche Sociali, Consiglio Nazionale delle Richerche (IRPPS-CNR), Roma, Italia. Università degli studi di Roma La Sapienza, Roma, Italia.

Marco Cellini: è assegnista di ricerca presso l'Istituto per la Ricerca sulla Popolazione e le Politiche Sociali (IRPPS) del Consiglio Nazionale delle Ricerche (CNR). Ha conseguito il Dottorato di Ricerca in Scienze Politiche presso l'Università LUISS Guido Carli di Roma. I suoi interessi di ricerca si concentrano sulla teoria democratica, sulla disuguaglianza economica e di genere, sulle politiche europee in materia di asilo e sulla governance globale.

Maria Cristina Antonucci, PhD in Sociologia politica (Università di Roma La Sapienza), è Ricercatrice in Scienze Sociali presso l'Istituto di Ricerche sulla Popolazione e le Politiche Sociali (IRPPS) del Consiglio Nazionale delle Ricerche (CNR) e professore a contratto in Comunicazione politica presso l'Università La Sapienza di Roma. I suoi interessi di ricerca si concentrano su lobbying e advocacy, governance urbana, partecipazione politica e sociale, questioni di genere, organizzazioni del terzo settore. 
Abstract: Negli ultimi decenni, il bilancio partecipativo (BP) è diventato uno degli strumenti più utilizzati e discussi della democrazia partecipativa. La letteratura teorica ed empirica sul BP ne hanno discusso e sottolineato i suoi punti di forza e i suoi limiti. I partigiani del BP hanno evidenziato la sua capacità di aumentare la partecipazione politica e di redistribuire i poteri politici a quei gruppi che di solito sono esclusi dagli spazi politici. I critici hanno messo in guardia sulla sua capacità di essere inclusiva e di mantenere le sue promesse di maggiore trasparenza e comunicazione da parte delle istituzioni e di effettiva partecipazione e responsabilizzazione dei cittadini coinvolti nel BP. La letteratura teorica, tuttavia, fa spesso riferimento alla democrazia partecipativa, in particolare al BP, come uno strumento in grado di risolvere quei problemi di mancanza di partecipazione e sfiducia nei confronti delle istituzioni democratiche che interessano i moderni stati liberali democratici. Il presente lavoro, attraverso l'analisi dei dati raccolti tra i partecipanti al BP del comun di Milano, si propone di tracciare un quadro del partecipante medio del BP e di capire in che misura il BP riesca a includere una sezione rappresentativa della Popolazione milanese. Confrontando le caratteristiche socio-demografiche dei partecipanti al BP, nonché la loro attitudine a partecipare alle attività politiche, con le caratteristiche dei residenti di Milano e dei cittadini italiani in generale, l'articolo rileva che i partecipanti al BP sono in media più anziani, più ricchi, più istruiti e politicamente più attivi rispetto alla media dei cittadini milanesi e alla media dei cittadini italiani, evidenziando come il BP di Milano non sia riuscito a coinvolgere fasce rappresentative della popolazione. Il documento si conclude sottolineando l'urgenza di riformare le pratiche di democrazia partecipativa per renderle più inclusive e quindi in grado di mantenere le promesse di risolvere i problemi che interessano le moderne democrazie rappresentative.

Parole chiave: democrazia partecipativa; Bilancio partecipativo; Partecipanti al budget partecipativo 


\section{Introduzione}

Il bilancio partecipativo $(\mathrm{BP})$ è uno degli strumenti più consolidati della democrazia partecipativa, nel contesto delle democrazie locali e urbane. Presenta una pluralità di virtù che spiegano l'utilizzo di questo strumento: i) è dotato di una storia consolidata, sin dal primo utilizzo a Porto Alegre nel 1989; ii) presenta una codificazione teorica ben strutturata e diffusa (Sintomer, Herzberg e Röcke 2016); iii) manifesta una specifica diffusione empirica di casi ed esempi su scala globale.

La plasticità del BP, adattabile alle più diverse realtà urbane (nei sistemi politici democratici e non (Sintomer et al.2012; Sintomer et al.2013; He 2011), e la sua polivalenza politica (Baiocchi e Ganuza 2014) hanno decretato il suo successo globale in un'epoca di profonda crisi per gli strumenti ed i canali tradizionali di partecipazione politica. La crisi della democrazia rappresentativa (Tormey 2014; Van der Meer 2017) ha aperto la prospettiva al BP come strumento di innovazione sociale e rinnovamento della partecipazione, spesso con il supporto di tecnologie che consentono la partecipazione democratica (Behl et al.2017).

Da un lato, gli studiosi che sostengono il BP come strumento e come pratica democratica sottolineano il suo contributo nella redistribuzione del potere politico ai gruppi esclusi all'interno delle democrazie (de Sousa Santos 1998); lo considerano come un meccanismo democratico per lo sviluppo locale/urbano (Cabannes 2004); sostengono la sua funzione di innovazione sociale nel processo decisionale locale/urbano (Novy e Leubolt 2005); contemplano la sua capacità di estendere la responsabilità istituzionale e promuovere l'efficacia dei diritti dei cittadini (Wampler 2010, 2012); e evidenziano la rilevanza nella costruzione di comunità (Lerner 2014, 2017). D'altra parte, gli studiosi critici rivelano una distinzione tra la dimensione puramente comunicativa del BP e la sua capacità di ampliare l'empowerment dei cittadini; sottolineano inoltre come il primo tenda a prevalere sul secondo (Baiocchi e Ganuza, 2014), mettendo in luce l'ambivalenza tra possibilità e limiti del BP quando quest'ultimo è 
considerato come unico metodo di democrazia partecipativa (Schugurensky 2009; Avritzer 2017).

La questione più impegnativa che si pone quando si studia il BP da una prospettiva empirica è capire in che misura, al netto della diffusione del modello BP (ora un gold standard emergente per la democrazia partecipativa a livello urbano), il bilancio partecipativo è efficace nelle sue funzioni di:

(1) Includere quei cittadini tradizionalmente esclusi o delusi dai circuiti tradizionali della democrazia rappresentativa, anche se solo in modo limitato e parziale, nei processi decisionali locali.

(2) Offrire reali possibilità di trasparenza e responsabilità per le istituzioni politiche e amministrative coinvolte nel processo di BP.

(3) Essere una pratica efficace di confronto democratico tra diverse posizioni e idee per i cittadini (sulla via di quella che Mansbridge 1999 e Talpin 2012 definiscono una " scuola di democrazia per le competenze dei cittadini), e non una mera attività procedurale volta a adempimenti burocratici.

(4) Incorporare nei processi decisionali e amministrativi delle istituzioni locali e cittadine coinvolte nel BP quella quantità di idee, proposte e orientamenti che sono espressi in modalità dal basso verso l'alto durante la procedura del BP.

Assumendo la posizione teorica di Baiocchi e Ganuza, sulla dimensione della comunicazione del BP che prevale sul lato coesistente dell'empowerment dei cittadini, questo lavoro esaminerà il caso di studio dei partecipanti al BP del comune di Milano, presentando i dati raccolti da un'indagine su questa pratica partecipativa. Questa prospettiva, basata sui dati sociodemografici e sugli atteggiamenti politici degli intervistati, potrebbe aiutare a capire se il BP fosse inteso come uno strumento per una migliore la comunicazione tra le istituzioni locali ei cittadini interessati a questo ambito di partecipazione o come meccanismo globale di 
empowerment democratico di tutti i cittadini, espandendo potenzialmente a chiunque gli spazi e gli strumenti del processo decisionale partecipativo nella città.

L'articolo si sviluppa come segue: la sezione 2 presenta una panoramica della letteratura scientifica più aggiornata sul $\mathrm{BP}$, introducendo la prospettiva teorica specifica di Baiocchi e Ganuza. La sezione 3 descrive brevemente l'esperienza del BP nella città italiana di Milano. La sezione 4 descrive i materiali e i metodi utilizzati nell'analisi. La sezione 5 presenta i risultati dell'analisi. La sezione, infine, 6 discute i risultati e presenta alcune osservazioni conclusive.

\section{Bilancio partecipativo nella letteratura scientifica: ricerca di una prospettiva per le sperimentazioni nelle città europee}

La letteratura scientifica sul BP manifesta una pluralità di contributi orientati a differenti finalità. Un filone teorico ha sviluppato l'approfondimento del contesto cognitivo della democrazia partecipativa, della sua capacità di innovazione, anche in relazione alla crisi degli strumenti della democrazia rappresentativa nell'era della diffidenza sistemica, del populismo e della crisi delle strutture tradizionali di rappresentanza (Tormey 2014, 2020). In questo campo di studio, il BP è considerato uno dei dispositivi di innovazione democratica utili a rinnovare il contesto dell'esercizio della partecipazione politica.

Una seconda linea di studi ha preso in considerazione la diffusione del BP come strumento empirico. Quest'area di analisi raccoglie e considera molteplici casi di studio, in diversi contesti politici democratici e non democratici, in cui il BP è stato impiegato con finalità corrispondenti alla logica delle istituzioni politiche, soprattutto locali, di coinvolgimento dei cittadini in formati di governance partecipativa, e la necessità dei cittadini di essere considerati attori delle scelte collettive nella vita della città e delle comunità locali. Da Porto Alegre (Abers et al., 2018) a New York (Jabola-Carolus et al.2020), da Medellin (Hajdarowicz 2018) a Madrid (Peña-López 2017), molte città hanno sperimentato, introdotto, implementato e valutato il BP come strumento integrativo della democrazia urbana, mentre molti contributi scientifici hanno 
riportato queste esperienze, tenendo conto dei rischi, delle opportunità, delle sfide e delle criticità di questi casi di studio, nelle città e nelle regioni.

Un terzo filone di studi si è concentrato sulla relazione tra i tipi di BP e le culture politiche in cui si sono sviluppate queste esperienze. Russon, Gilman e Wampler (2019) esaminano le differenze nella progettazione del BP in Nord America e in America Latina, facendo riferimento a un'analisi multifattoriale, includendo le variazioni nelle rispettive culture politiche. Nel contesto europeo è possibile richiamare i contributi di Sintomer, Yves, Carsten Herzberg e Anja Röcke (2008) e, più recentemente, di Sintomer, Herzberg e Röcke (2016), che centrano la loro analisi del ruolo e della funzione del $\mathrm{BP}$ in una dimensione continentale, tenendo in considerazione le esperienze locali e regionali alla luce del contesto politico e istituzionale di riferimento. Secondo questi autori, il BP in Europa si confronta con alcuni fattori sistemici: una crisi specifica di legittimità politica, diretta soprattutto verso le istituzioni rappresentative, la perdita di rapporto tra rappresentanti e rappresentati e un accentuato interesse per la dimensione partecipativa locale rispetto a quello nazionale.

In questa prospettiva, che interseca culture politiche, condizioni istituzionali e partecipazione civica, i tre autori individuano e caratterizzano sei linee di sviluppo del BP nelle esperienze che si sono succedute in Europa: democrazia partecipativa; democrazia di prossimità, modernizzazione partecipativa; partecipazione multi-stakeholder; neocorporativismo e sviluppo della comunità. Le sei linee di sviluppo costituiscono punti di riferimento ideali (per natura, condizioni, orientamento e modello di sviluppo di questo strumento partecipativo) qualificati per identificare le esperienze specifiche che avvengono nei contesti delle città e delle aree di applicazione della BP in Europa.

Un quarto filone di ricerca ha sviluppato lo studio del rapporto tra nuove tecnologie ICT e BP. Questa linea di studi ha evidenziato la relazione tra le tecnologie abilitanti, già utilizzate nella vita quotidiana per lo svolgimento di attività nelle istituzioni e nelle amministrazioni, e lo 
sviluppo della dimensione partecipativa attraverso tali dispositivi informatici e telematici (Kamal et al.2016; Cardoso Sampaio 2016; Parra et al.2017), spesso collegando tecnologie e BP nel contesto di studi sulle 'città intelligenti' (Effing e Groot 2016).

Infine, una quinta area di studio considera il BP come il principale strumento per l'affermazione di nuove modalità di democrazia partecipativa, con un approccio critico. In quest'area di analisi si segnala il contributo di Baiocchi e Ganuza $(2014,2016)$ che mette in luce le aporie e le incertezze del BP nel raggiungimento di una maggiore trasparenza e una migliore comunicazione da parte delle istituzioni, e l'effettiva partecipazione e responsabilizzazione dei cittadini che sono coinvolti nel BP. Secondo questa visione, è possibile suddividere gli effetti della BP in termini: da un lato, di maggiore comunicazione tra cittadini e istituzioni decisionali locali, e, dall'altro, di maggiore impatto sulla capacità dei cittadini di informarsi apprendere, partecipare, negoziare, sintetizzare e deliberare all'interno dei percorsi stabiliti dal BP. Mentre il primo effetto "comunicativo" è spesso possibile nel processo del BP, l'empowerment partecipativo dei cittadini spesso non è particolarmente efficace in tali processi.

Quest'ultimo contributo critico sembra adattarsi in modo particolarmente efficace al BP di Milano. Da un lato, il livello di comunicazione tra le istituzioni cittadine che hanno proposto il BP e i cittadini coinvolti nel processo hanno trovato una sicura espansione, anche grazie ai dispositivi multicanale per l'accesso alle procedure previste dal BP. Dall'altro, invece, dai dati raccolti dalla nostra indagine, lo spazio per l'empowerment dei cittadini è apparso circoscritto ad una fascia specifica della popolazione cittadina: quella ad alto capitale economico, sociale e culturale e già orientata verso un'elevata partecipazione politica e sociale, secondo le forme tradizionali previste dal sistema politico. Non si può parlare pienamente di empowerment nella partecipazione se non si amplia la base dei cittadini coinvolti in queste pratiche innovative, senza distinzione di reddito, istruzione e livello di partecipazione alla vita pubblica. In questo, 
nelle conclusioni, verrà riconsiderata la prospettiva di Baiocchi e Ganuza, per discutere il senso dei risultati emersi dall'analisi dei dati sul BP di Milano.

\section{L'esperienza del Bilancio Partecipativo di Milano: contesto di ricerca}

La città di Milano è il secondo comune italiano per numero di cittadini ed è il capoluogo di una delle regioni italiane più ricche: la Lombardia. Secondo i dati forniti dall'Istituto Nazionale di Statistica, a gennaio 2020 la popolazione del Comune di Milano ammontava a 1.396.059 cittadini (ISTAT 2020), rappresentando il 2,32\% della popolazione italiana. Essendo aperto alla partecipazione di tutti quei soggetti che hanno un rapporto continuativo con il territorio comunale per motivi di studio, lavoro o abitativo, e almeno 16 anni, i potenziali partecipanti al BP sono 1.207.527.

Il Bilancio partecipativo è stato introdotto nel Comune di Milano a partire dal 2015 con la prima edizione dell'iniziativa ed è stato impostato in modo più sistematico nel biennio 2017-2018. Nella proposta fatta ai cittadini di Milano, il BP è stato inteso come uno strumento di amministrazione partecipativa che, sulla base di un budget stanziato dal Comune, permetteva direttamente ai cittadini di proporre, programmare e votare interventi per il proprio territorio, che verranno poi realizzati dalla città. L'approccio originale al BP, nella prima fase dal 2015 al 2017/8, è sintetizzato dalle parole dell'Assessore ai Lavori Pubblici di Milano Carmela Rozza: "La città deve continuare ad essere aperta. L'impegno del Comune, anche nella prossima legislatura, sarà quello di continuare a riqualificare gli spazi aperti e condivisi della città e rendere strutturale il bilancio partecipativo" (Giannattasio 2016).

Il BP si pone come strumento dedicato alla riqualificazione urbana, con aperture alla partecipazione di associazioni e cittadini. Solo in una seconda fase del progetto, con la designazione di un Assessore alla Partecipazione, Lorenzo Lipparini, il bilancio partecipativo inizia il suo percorso come strumento di democrazia partecipativa urbana, e non più solo come mezzo di rigenerazione cittadina. Nelle parole dell'Assessore Lipparini, espresse nel corso di 
un evento speciale sulla partecipazione a Milano, le esperienze svolte nello studio BP "mostrano come i cittadini siano coinvolti dal Comune nella sperimentazione di percorsi partecipativi di qualità e nuove modalità di amministrazione condivisa dei beni comuni, mentre aumentare il perimetro dei diritti di cittadinanza" (Dazzi 2019). In questa nuova, prospettiva del BP, l'amministrazione milanese evidenzia come il bilancio partecipativo non sia più solo uno strumento riservato alla rigenerazione urbana, ma rappresenti un mezzo innovativo per ampliare la democrazia partecipativa in una serie di ambiti di politica urbana.

Così, da strumento riservato ad una specifica area di policy, lo standard del BP si sposta, nelle intenzioni della città di Milano, diventando dispositivo di democrazia partecipativa, per garantire un ampliamento degli strumenti partecipativi ai cittadini e per costruire un nuovo modello di governance partecipativa per le diverse aree della politica urbana.

Secondo quanto espresso dal Consiglio Comunale nella Delibera 983 approvata il $1^{\circ}$ giugno 2017, l'obiettivo politico del BP è stato quello di avvicinare i cittadini alle istituzioni e aumentare il loro senso di appartenenza collettiva. In questo senso il BP è stato concepito per il co-design delle politiche pubbliche da parte della pubblica amministrazione e della società civile, nei limiti di un budget prestabilito.

Il processo del BP si compone di due macro-fasi (disciplinate per ruoli e responsabilità dal Contratto di Partecipazione) quella deliberativa e quella attuativa. Con la prima fase la pubblica amministrazione raccoglie le proposte prodotte dall'autonoma iniziativa dei cittadini, e dagli organismi intermedi, selezionando quelle meritevoli di realizzazione mediante votazione. Con la seconda fase, la Pubblica Amministrazione attua le proposte premiate dai cittadini, operando, per motivi di trasparenza e responsabilità, sotto il controllo diretto e permanente dei cittadini stessi, affinché l'intero processo possa costituire un'esperienza comunitaria (Comune di Milano 2017). Il monitoraggio dei progetti da parte dei cittadini consente di seguirne l'andamento temporale lungo il tracciato amministrativo e tecnico, di consultare i documenti prodotti, di 
rimanere aggiornati sull'avvio dei lavori nei vari municipi. Da maggio 2018 è in corso la fase di monitoraggio che segue lo sviluppo del progetto, dallo staff di Comunicazione al cantiere dei progetti.

Per quanto riguarda l'entità dell'investimento nel BP, è importante sottolineare che a fronte di un totale di 2.734.365.781,36 euro di spese correnti effettuate dal Comune di Milano nel 2018, il budget messo a disposizione per l'iniziativa è stato pari a 4.500.000,00 euro, ovvero solo lo $0,164 \%$ delle spese totali.

Per quanto riguarda le modalità di partecipazione, ogni cittadino aveva diritto di presentare un progetto riguardante una qualsiasi area di intervento tra quelle di competenza comunale, tuttavia c'erano una serie di regole relative a quale tipo di progetto fosse ammissibile o meno: le aree territoriali, edilizie o tematiche sono di competenza dei Comuni e/o del Comune, non di privati; il progetto doveva prevedere solo opere pubbliche ed eventualmente l'acquisto di beni durevoli; non erano ammessi progetti che comportassero l'acquisto di servizi, consulenze, attività professionali; il valore stimato dell'investimento doveva essere compreso tra 100.000 euro e 500.000 euro, incluso l'IVA e tutti i costi accessori per la realizzazione; l'intervento non doveva essere in conflitto con progetti preesistenti o in corso di progettazione o con strumenti di pianificazione territoriale già approvati; l'investimento non doveva essere antieconomico o contrario ai principi di efficienza, efficacia e sostenibilità (Comune di Milano 2017).

Per quanto riguarda il BP 2015, sono stati proposti, votati, selezionati e realizzati 16 progetti nelle categorie: ambiente, economia, famiglia e giovani, inclusione sociale, rigenerazione urbana, scuola, sicurezza, strade e mobilità, tecnologia, sport e tempo libero. Nell'ambito del BP 2017/18, nelle stesse aree di intervento, sono stati proposti 47 progetti e ne sono stati selezionati 12 , le cui fasi costruttive sono ancora in corso.

Dei circa 1.207.527 residenti di Milano che avevano diritto a partecipare al processo del BP, solo circa 17.000 individui hanno deciso di prendere parte all'iniziativa. Ciò significa che il BP 
di Milano è riuscito a coinvolgere solo una relativa minoranza, che rappresenta l'1,41\% della popolazione comunale avente diritto a partecipare. Questi dati da soli potrebbero mettere in discussione l'effettiva capacità della democrazia partecipativa di risolvere i problemi che affliggono le moderne democrazie rappresentative, tuttavia, per fare una seria valutazione sull'efficacia dei processi del BP, è importante valutare non solo l'entità della partecipazione ma anche le sue tipologie.

\section{Materiali and metodi}

Per valutare il profilo del partecipante medio al BP, e per capire fino a che punto esso è rappresentativo o meno della cittadinanza italiana in generale e della popolazione di Milano in particolare, nell'ambito del progetto Milano Partecipa (Cellini e Antonucci 2020), abbiamo amministrato un sondaggio online a un campione dei cittadini che hanno partecipato all'edizione 2018 del BP.

Il sondaggio è stato somministrato online, tramite l'utilizzo del software Limesurvey, inviando il link del sondaggio alle 17.000 persone che si sono registrate al portale del BP. La compilazione del questionario è stata aperta dal 10 dicembre 2020 al 29 febbraio 2020.

Su 17.000, 683 partecipanti hanno deciso di completare il questionario. Il nostro campione, quindi, rappresenta solo il 4,01 per cento dell'universo di riferimento, tuttavia, il calcolo dell'errore di campionamento per le variabili demografiche rilevanti (sesso, livello di istruzione e classe di età) ha mostrato che con un livello di confidenza del $99 \%$ tali errori ammontano rispettivamente soltanto a $0,050,0,196$ e 0,088 , a conferma che il campione è rappresentativo dell'universo di riferimento.

Dal questionario abbiamo recuperato le seguenti informazioni sui partecipanti al BP: età, livello di reddito, livello di istruzione, condizione professionale, partecipazione alle elezioni locali, nazionali ed europee, se sono membri di un partito politico e se hanno partecipato a una 
manifestazione e ad attività di volontariato nell'anno precedente la somministrazione del questionario.

Per valutare se i partecipanti al BP sono rappresentativi della popolazione italiana in generale e della popolazione di Milano in particolare, abbiamo raccolto le stesse informazioni per i due gruppi. I dati sui residenti del comune di Milano e sui cittadini italiani sono stati recuperati da più fonti. È importante sottolineare che per le adesioni ai partiti e per la partecipazione alle attività di volontariato e alle manifestazioni i dati sono stati raccolti e confrontati solo per i cittadini italiani in quanto non disponibili per i residenti Milanesi.

I dati su età, reddito, livello di istruzione e condizione occupazionale dei cittadini italiani e Milanesi provengono dall'ISTAT (2020) e si riferiscono all'anno 2019. I dati sono stati ricodificati per essere confrontati con quelli raccolti attraverso la nostra indagine. I dati sull'appartenenza ai partiti politici e sulla partecipazione alle attività di volontariato provengono dal database World Value Survey, Wave 5, e si riferiscono agli anni 2005-2009. Infine, i dati sulla partecipazione alle dimostrazioni provengono dal database European Social Survey, Wave 9, e si riferiscono all'anno 2018.

\section{Resultati: Valutare chi partecipa al bilancio partecipativo}

Nell'ultimo decennio c'è stato un vivace dibattito tra accademici e professionisti sulla necessità e l'opportunità di aumentare le pratiche partecipative a livello locale. Tuttavia, da un punto di vista concettuale, l'opportunità di utilizzare metodi partecipativi come il BP dovrebbe considerare la capacità di includere, da un lato, quanti più cittadini possibile nel processo partecipativo e, dall'altro, di includere cittadini rappresentativi della popolazione del contesto in cui viene eseguito il processo. Altrimenti, c'è il rischio che i processi partecipativi possano diventare un ulteriore strumento utilizzato da alcuni gruppi o élite per perseguire i propri interessi particolari (Uddin, Mori e Adhikari 2019). È importante sottolineare che il BP, così come le altre forme esistenti di democrazia partecipativa e deliberativa, non dovrebbe sostituire 
gli organi politici dei contesti in cui è implementata né essere impiegata in tutti i processi decisionali, ma piuttosto dovrebbe integrarli consentendo ai cittadini di intervenire nei processi decisionali relativi a un insieme limitato e specifico di aspetti politici.

Per valutare se e in che misura i cittadini che hanno partecipato all'esperienza del BP a Milano siano rappresentativi del contesto socio-economico locale e nazionale, è necessario dunque confrontare i dati raccolti attraverso l'indagine con i corrispondenti dati locali e nazionali.

La Figura 1 descrive la struttura dell'età dei partecipanti al BP rispetto alla struttura dell'età dei residenti di Milano e dei cittadini italiani. È interessante notare che mentre la struttura dell'età dei cittadini italiani è abbastanza simile a quella dei residenti nel comune di Milano, entrambe differiscono sostanzialmente da quella dei partecipanti al BP. Dalla partecipazione alla BP sono quasi esclusi i cittadini dai 16 ai 29 anni (che rappresentano il 13,6 per cento dei residenti milanesi e il 14,1 per cento dei cittadini italiani), che rappresentano solo il 3,7 per cento del campione. Allo stesso tempo, i cittadini con più di 40 anni sono l'84,8 dei partecipanti al BP, rappresentando in eccesso sia i residenti di Milano che i cittadini italiani che rappresentano il 59,2 per cento e il 60,5 per cento delle rispettive popolazioni. 
Figura 1. Confronto tra la struttura per età dei partecipanti al Bilancio Partecipativo e la relativa struttura per età dei residenti a Milano e dei cittadini italiani.

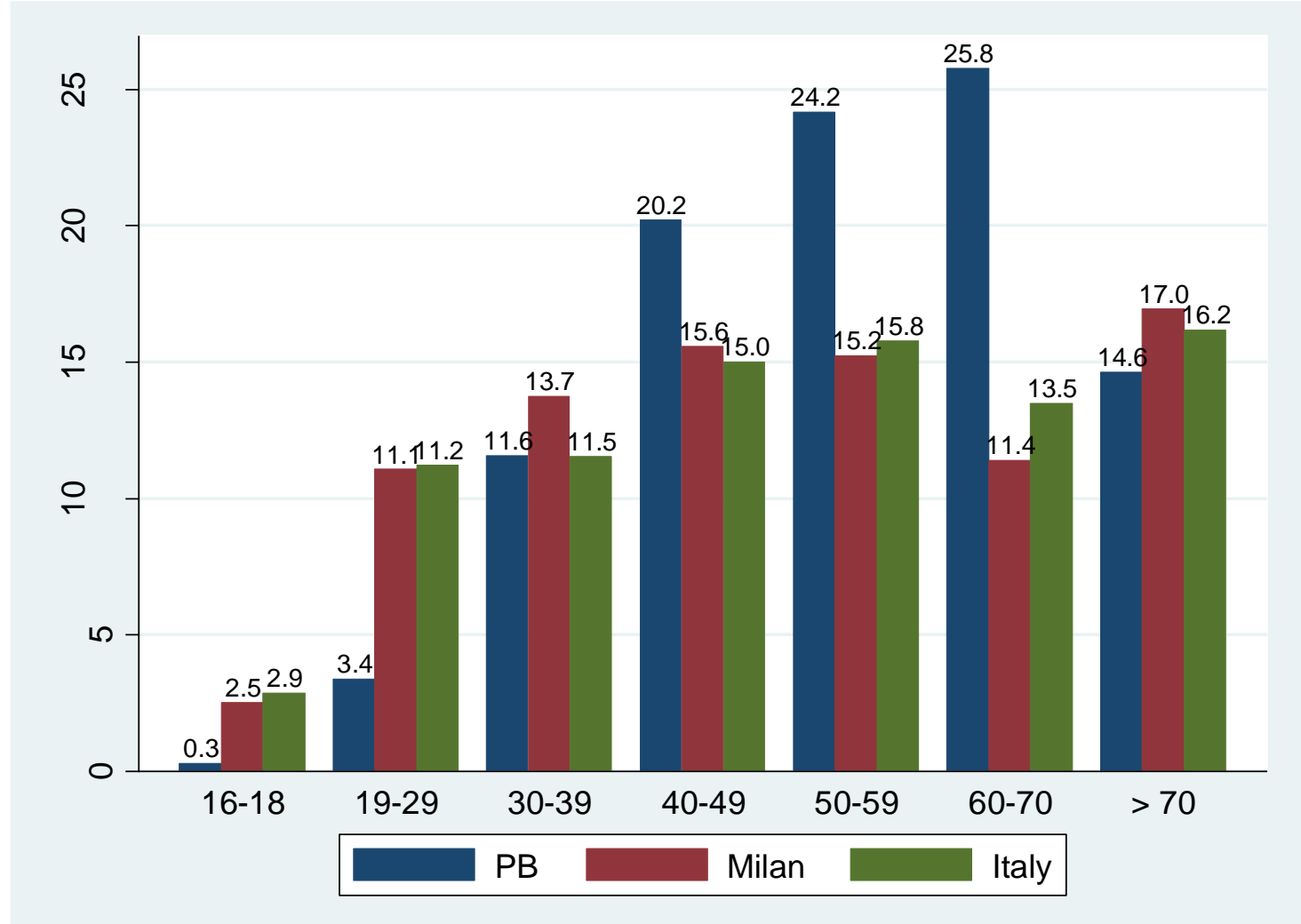

Fonte: dati ISTAT (2020) per residenti a Milano e cittadini italiani

La Figura 2 mostra graficamente il confronto tra il reddito annuo dei partecipanti al BP e quello dei milanesi e dei cittadini italiani nel complesso. La figura mostra due dati interessanti. Da un lato, la maggioranza relativa dei partecipanti al BP, dei residenti a Milano e dei cittadini italiani (rispettivamente 62,5 per cento, 52,1 per cento e 50 per cento) rientra nella fascia di reddito da 15.001 a 55.000 euro. Dall'altro lato, tuttavia, il campione dei partecipanti al BP si discosta fortemente dai dati locali e nazionali per quanto riguarda i livelli di reddito bassi e alti. Infatti, mentre la percentuale di partecipanti al BP che dichiara redditi compresi tra 0 euro e 15.000 euro è 5,5, i residenti di Milano e cittadini italiani che dichiarano lo stesso guadagno sono rispettivamente il 35,3 per cento e il 44,5 per cento della corrispondente popolazione. Allo stesso modo, dall'altra parte della scala dei redditi, i partecipanti al BP che guadagnano più di 
75.000 euro rappresentano il 15,4 per cento del campione contro il 7,6 per cento e il 2,2 per cento dei milanesi e dei cittadini italiani.

Figura 2. Confronto dei guadagni tra partecipanti al Bilancio Partecipativo, residenti a Milano e cittadini italiani

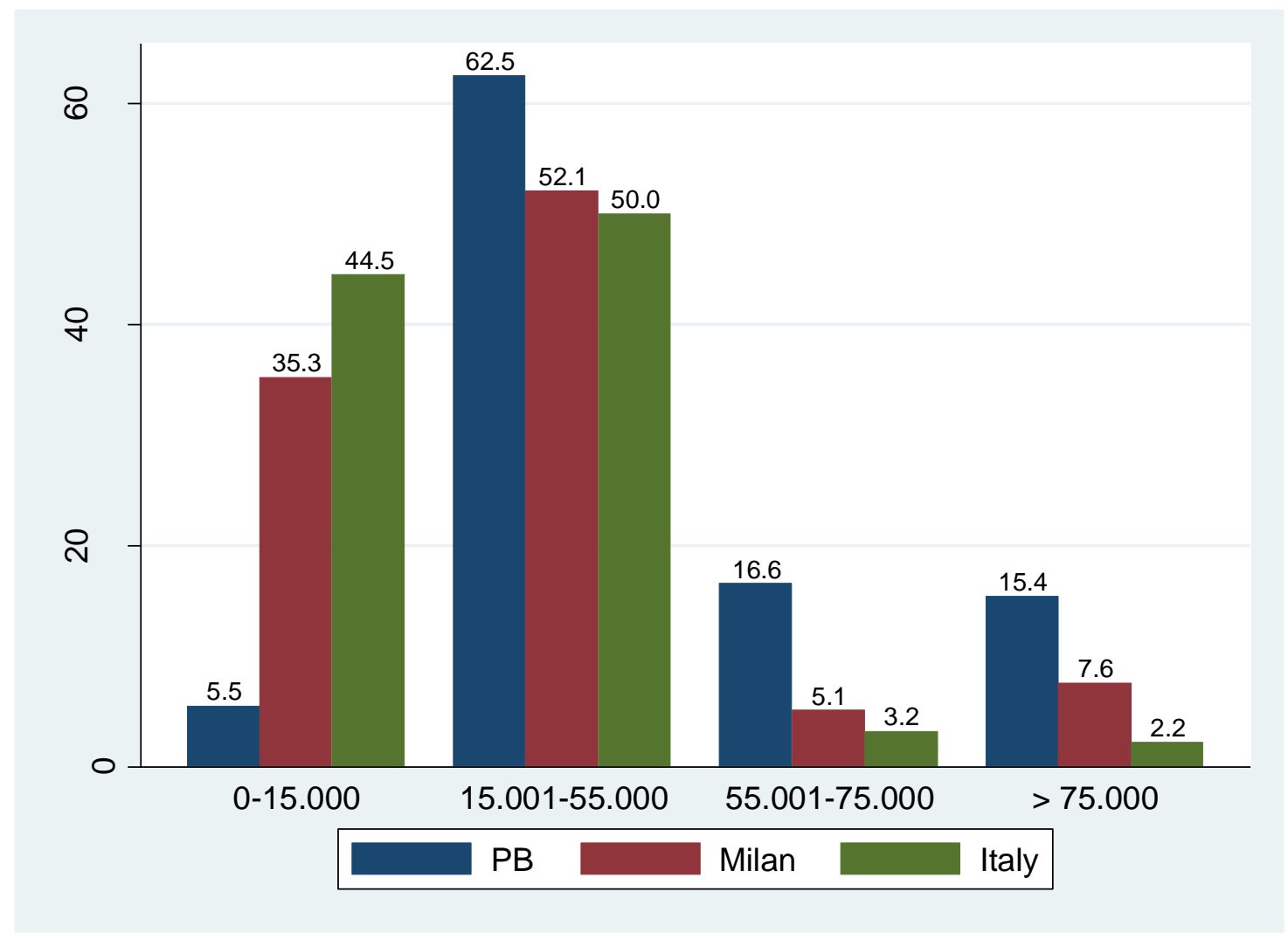

Fonte: dati ISTAT (2020) per residenti a Milano e cittadini italiani

La Figura 3 mostra il confronto del livello di istruzione dei partecipanti al BP, dei residenti milanesi e dei cittadini italiani. Il dato mostra che rispetto ai residenti milanesi e ai cittadini italiani nel complesso, i partecipanti al BP hanno un livello medio di istruzione più elevato. In particolare, i dati dei partecipanti al BP divergono in modo consistente nei due estremi della scala educativa. Mentre i residenti di Milano e i cittadini italiani che non hanno alcuna istruzione o hanno soltanto un'istruzione elementare rappresentano rispettivamente 1 '8,7\% e il 16,2\% della popolazione, i partecipanti al BP rappresentano solo lo 0,2\% del campione. Dall'altra parte della scala, i milanesi e i cittadini italiani con istruzione terziaria o post-terziaria rappresentano rispettivamente il $26,2 \%$ e il $15 \%$ della popolazione, mentre i partecipanti al BP rappresentano il $60,2 \%$ del campione. 
Figura 3. Livello di istruzione a confronto tra partecipanti al Bilancio Partecipativo, residenti a Milano e cittadini italiani

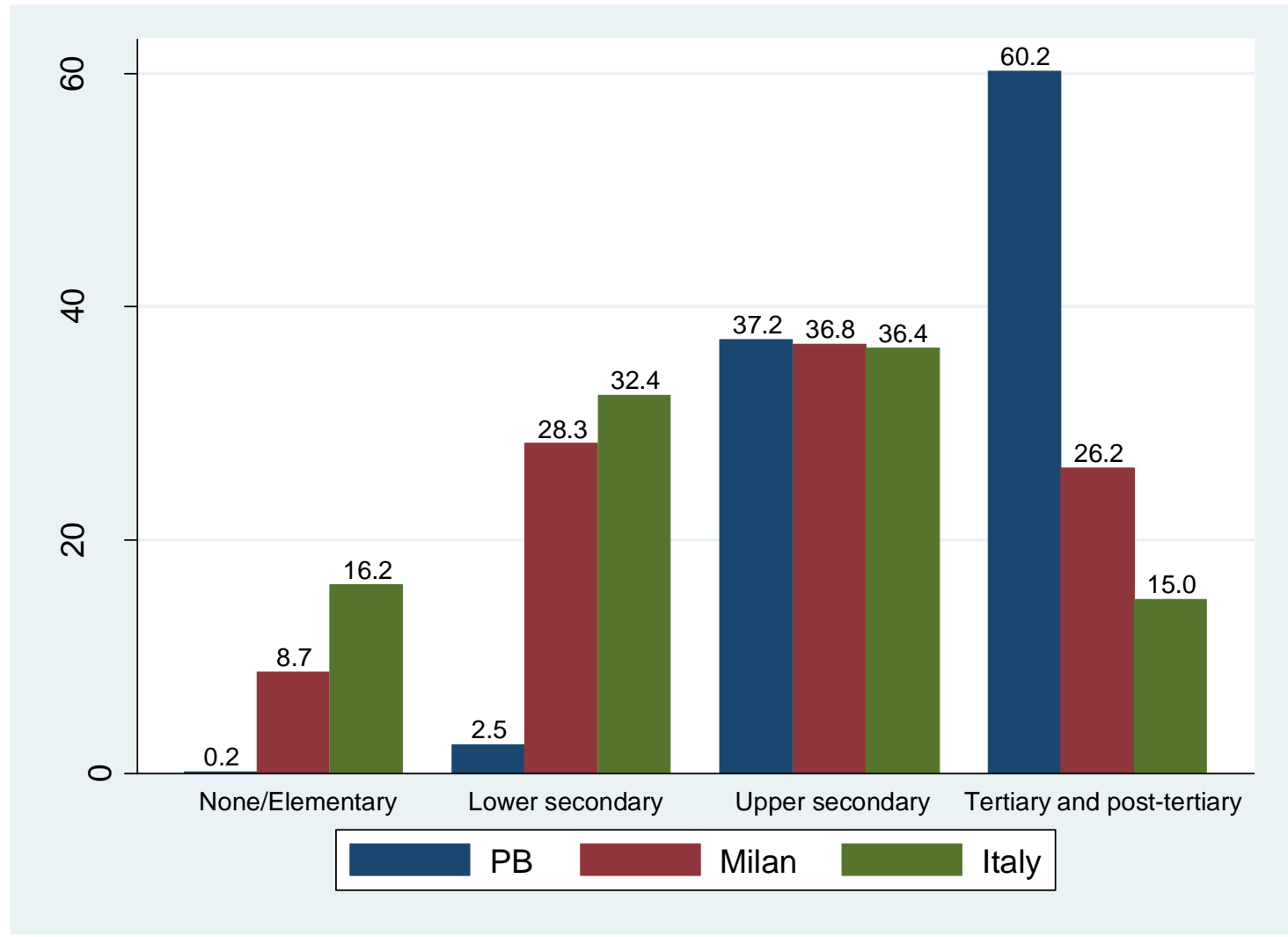

Fonte: dati ISTAT (2020) per residenti a Milano e cittadini italiani

Un'immagine diversa appare guardando i dati riguardanti l'occupazione dei partecipanti al BP

(Figura 4). Il confronto dei loro dati con quelli dei milanesi e dei cittadini italiani evidenzia alcune differenze tra i partecipanti al BP e i residenti milanesi, mentre mostra una sostanziale convergenza tra i primi e cittadini italiani. Rispetto ai dati milanesi e italiani, i partecipanti al BP hanno mostrato una quota inferiore di disoccupati, casalinghe e studenti, e una quota maggiore di occupati e pensionati. 
Figura 4. Confronto della condizione occupazionale tra partecipanti al Bilancio Partecipativo, residenti a Milano e cittadini italiani

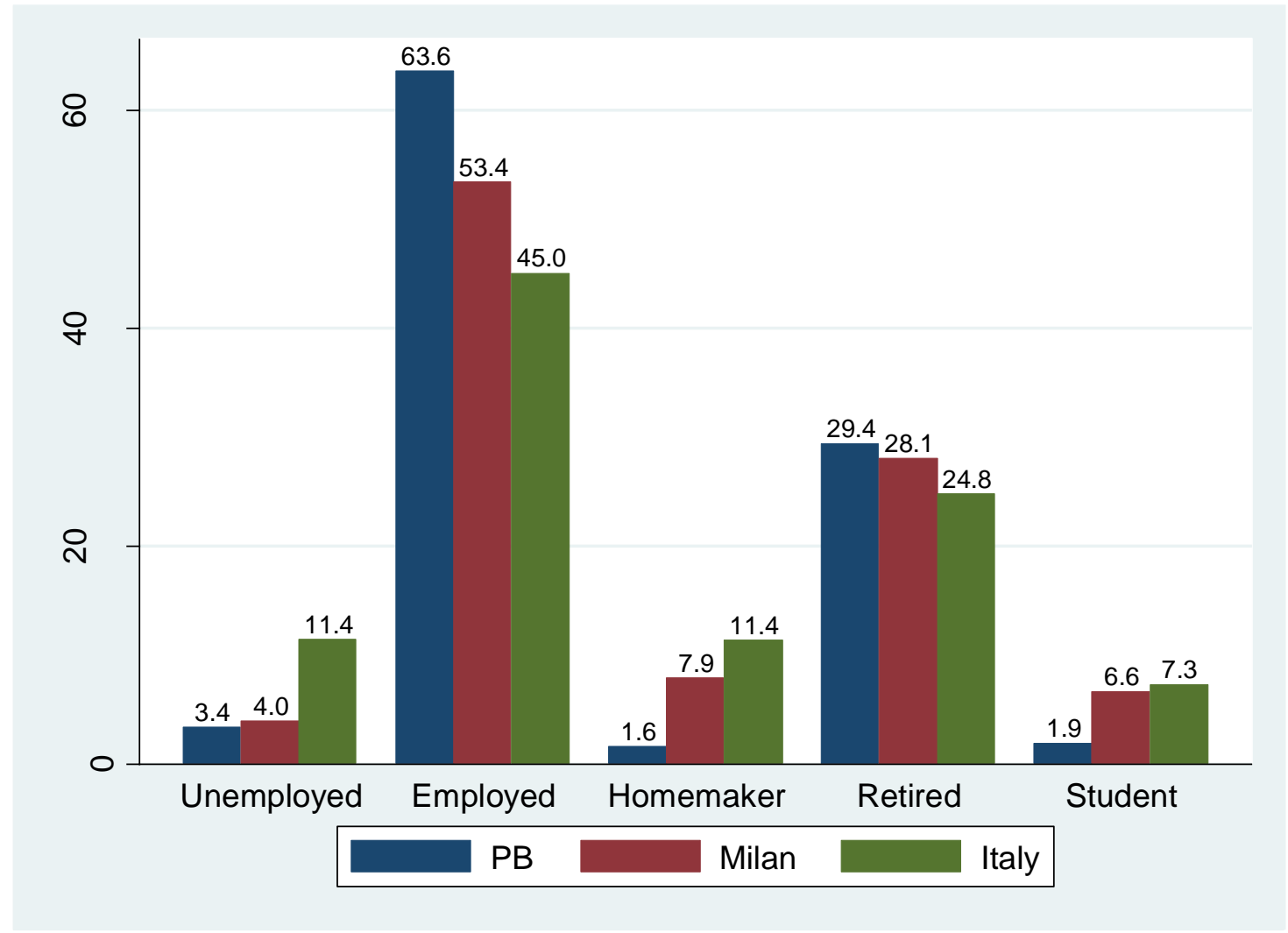

Fonte: dati ISTAT (2020) per residenti a Milano e cittadini italiani

Infine, il confronto sull'affluenza alle elezioni locali, nazionali ed europee tra i partecipanti al BP, i residenti di Milano e i cittadini italiani (Figura 5) mostra che mentre i dati di Milano e quelli italiani sono sostanzialmente convergenti, rispettivamente il 63,75\% e il 67,35\%, l'affluenza alle urne dei partecipanti al BP è molto superiore a entrambi con una quota del 94,69 per cento. 
Figura 5. Confronto affluenza locale, nazionale ed europea tra partecipanti al Bilancio Partecipativo, residenti a Milano e cittadini italiani

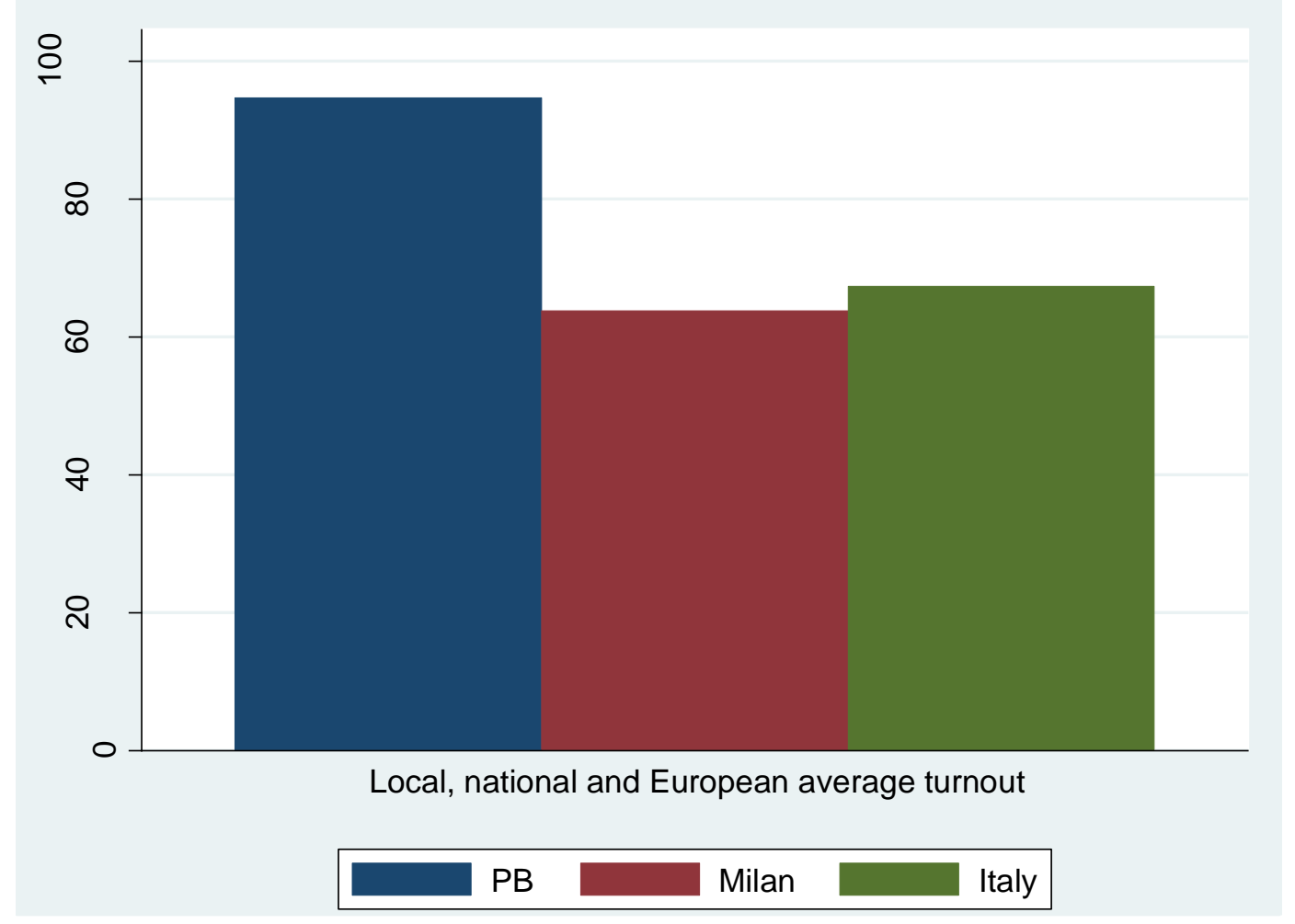

Fonte: dati su residenti a Milano e cittadini italiani raccolti dal Ministero dell'Interno (2020)

Questi risultati sono parzialmente rispecchiati anche dalla partecipazione dei partecipanti al BP alle attività sociali e politiche. La Figura 6 riporta graficamente il confronto tra cittadini italiani e i partecipanti al BP rispetto alla partecipazione ad attività di volontariato e a manifestazioni, e alla membership di partiti politici. I dati mostrano due risultati interessanti: da un lato, i partecipanti al BP partecipano in media in maniera superiore ad attività di volontariato e manifestazioni politiche ma, dall'altro, la quota di partecipanti al BP che sono membri di un partito è inferiore alla quota nazionale. 
Figura 6. Confronto tra cittadini italiani e partecipanti al BP che partecipano ad attività di volontariato, che fanno parte di un partito e che partecipano a manifestazioni

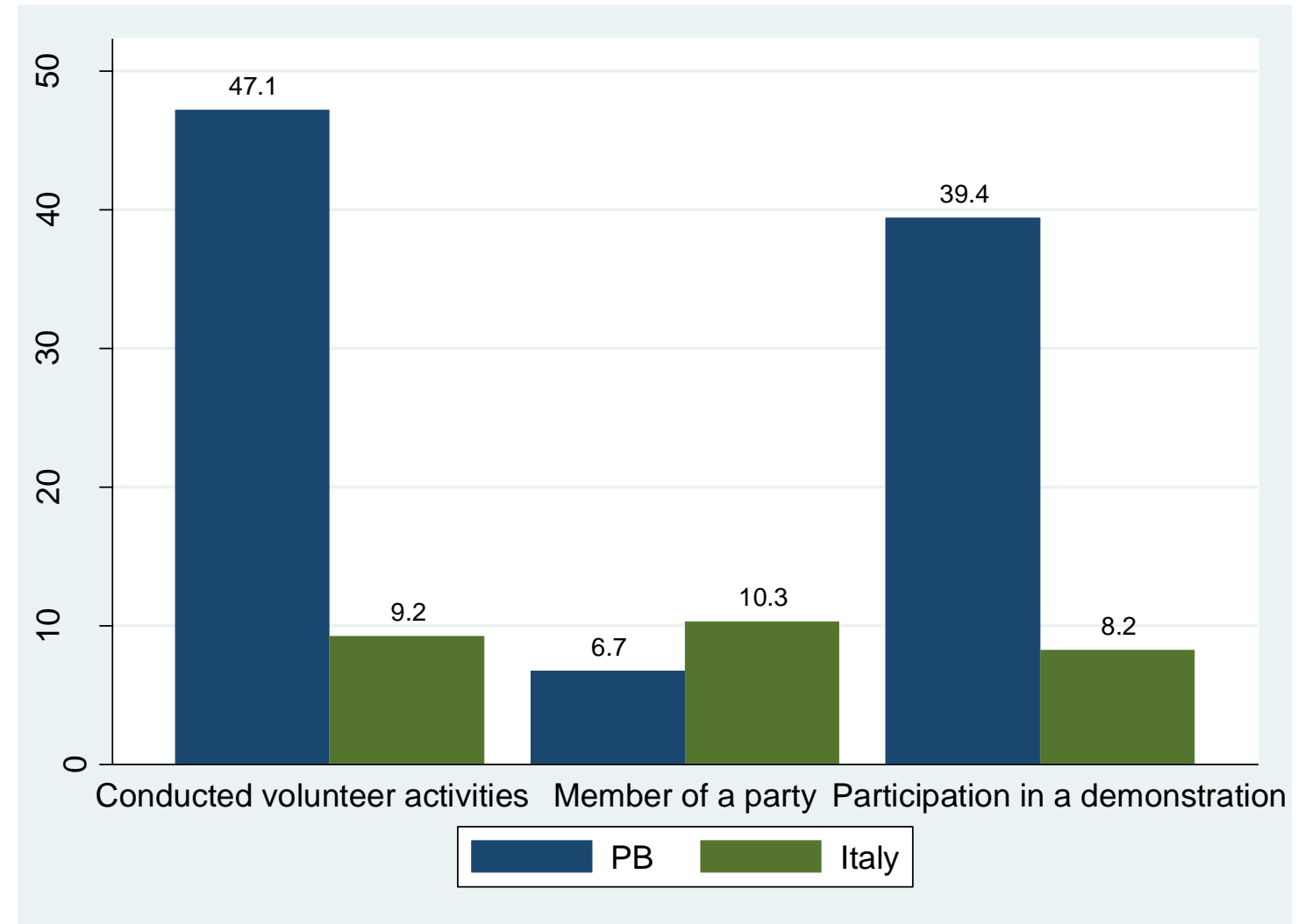

Fonte: i dati sui cittadini italiani sono tratti dal World Value Survey (2010) per l'appartenenza al partito e le attività di volontariato; e da European Social Survey (2018) per la partecipazione a manifestazioni.

\section{Discussione and conclusioni}

Attraverso l'analisi dei dati raccolti nell'ambito del progetto MIPAR, questo lavoro ha mirato a un duplice obiettivo: da un lato disegnare il profilo socio-economico dei partecipanti al BP di Milano e, dall'altro, rispondere alla seguente domanda di ricerca: in che misura il bilancio partecipativo a Milano è riuscito a coinvolgere un campione rappresentativo della popolazione, offrendo loro adeguate opportunità di partecipazione a livello cittadino ai processi decisionali su temi specifici (ambiente, economia, famiglia e giovani, inclusione sociale, rigenerazione urbana, scuola, sicurezza, strade e mobilità, tecnologia, sport e tempo libero), sostenendo la comunicazione tra cittadini e istituzioni e stimolando l'empowerment dei cittadini nella democrazia urbana. 
La questione della rappresentatività delle pratiche partecipative è spesso trascurata mentre dovrebbe essere centrale nelle discussioni che crescono intorno alla democrazia partecipativa e deliberativa. La letteratura sulla democrazia partecipativa e deliberativa, infatti, descrive tali metodi come una soluzione teoricamente ed empiricamente valida per rinnovare le democrazie rappresentative (Tormey 2014, 2020; Kersting et al.2016) e per risolvere i problemi legati al metodo rappresentativo: bassa partecipazione politica e sfiducia verso le istituzioni politiche e democratiche (Hooghe e Marien 2013).

I benefici promessi dai metodi democratici partecipativi e deliberativi (Barge 2002; Cini 2011), tuttavia, potrebbero essere raggiunti se, e solo se, tali metodi fossero in grado di coinvolgere settori ampi e rappresentativi della popolazione in cui sono implementati. Altrimenti rischiano di replicare solo i problemi impliciti nella democrazia rappresentativa tradizionale, su una scala diversa.

Per rispondere alla domanda proposta, abbiamo analizzato i dati raccolti tra un campione di partecipanti al BP di Milano, tracciando il profilo del partecipante medio e confrontando poi i dati raccolti con quelli relativi all'intera popolazione del comune di Milano e alla popolazione italiana nel suo complesso. I risultati dell'analisi mostrano come il BP di Milano non sia riuscito a coinvolgere una sezione rappresentativa della popolazione milanese, né una sezione rappresentativa della cittadinanza italiana.

In primo luogo, i dati mostrano che i giovani cittadini (16-20 anni), che rappresentano il 13,6 per cento della popolazione di Milano e il 14,1 per cento della cittadinanza italiana, sono quasi esclusi dalla partecipazione al BP, rappresentando solo il 3,7 per cento del campione. In senso opposto, i cittadini tra i 40 ei 70 anni risultano essere sovra rappresentati rispetto sia alla popolazione milanese che a quella italiana. I dati, quindi, dimostrano che il bilancio partecipativo di Milano non riesce a coinvolgere la fascia più giovane della popolazione 
risultata sottorappresentata rispetto ai contesti locale che nazionale, mentre ha coinvolto un campione sovra rappresentativo di individui nella fascia di età 40-70 anni.

In secondo luogo, dall'analisi della situazione economica dei partecipanti emergono due risultati interessanti: da un lato, la maggior parte dei partecipanti al BP ha riportato un reddito annuo compreso tra 15.001 e 55.000 euro, in linea con i milanesi e con i cittadini italiani nel complesso; d'altra parte, il reddito annuale dei partecipanti al BP diverge in modo significativo quando si considerano i redditi più bassi e quelli più alti. Sotto questo aspetto, infatti, i redditi inferiori rappresentano solo una piccola minoranza dei partecipanti al BP (5,5 per cento) mentre rappresentano il 35,3 per cento dei residenti di Milano e il 44,5 per cento dei cittadini italiani, mentre i redditi superiori a 55.000 euro sono sovra rappresentati all'interno dei partecipanti al BP che rappresentano il 32 per cento contro il 12,7 per cento e il 5,4 per cento rispettivamente dei milanesi e dei cittadini italiani. Il confronto dei redditi, quindi, ha mostrato come i residenti a basso reddito siano relativamente quasi esclusi dalla partecipazione al BP milanese. Questo dato è particolarmente rilevante perché evidenzia come i residenti indigenti ed emarginati tendano a non prendere parte alle attività partecipative, che al contrario potrebbero essere utilizzate dal Comune per rafforzare il rapporto con i residenti più vulnerabili.

In terzo luogo, l'analisi dei livelli di istruzione rivela come i partecipanti al BP siano in media iper-educati rispetto ai residenti di Milano e ai cittadini italiani. Questo confronto rafforza la preoccupazione circa la rappresentatività dei partecipanti al $\mathrm{BP}$, mostrando che le persone con un basso livello di istruzione tendono a non partecipare. Questo può essere il sintomo della difficoltà intrinseca del processo stesso e deve essere considerato per rendere il processo più inclusivo e rappresentativo.

In quarto luogo, i dati sullo stato occupazionale hanno mostrato come rispetto ai milanesi, $\mathrm{i}$ partecipanti al BP abbiano una quota inferiore di disoccupati e una quota maggiore di casalinghe e studenti, mentre rispetto ai dati nazionali mostrano un livello inferiore di casalinghe e 
disoccupati. Nel complesso, per quanto riguarda lo stato occupazionale i dati evidenziano una certa capacità del BP di includere diverse categorie lavorative, con la notevole eccezione dei residenti disoccupati che sono risultati sottorappresentati rispetto sia ai milanesi che ai cittadini italiani.

Infine, l'analisi della partecipazione politica evidenzia come i partecipanti al BP siano più attivi dei residenti di Milano e dei cittadini italiani, segnalando un più alto grado di partecipazione alle elezioni locali, nazionali ed europee. Un segno parzialmente diverso, tuttavia, appare quando si considera il confronto tra la partecipazione ad attività di volontariato e a manifestazioni politiche, nonché rispetto all'appartenenza a un partito politico. A questo proposito, infatti, i dati mostrano da un lato che i partecipanti al BP tendono a partecipare ad attività di volontariato e a manifestazioni politiche in misura maggiore rispetto ai residenti milanesi e ai cittadini italiani, mentre dall'altro lato essi tendono a non essere coinvolti nelle adesioni ai partiti politici.

Riassumendo, i risultati della nostra analisi mostrano come, confrontando le caratteristiche dei partecipanti al BP milanese con quelle dei residenti milanesi e dei cittadini italiani, emerge come il BP implementato a Milano non riesca a includere una sezione rappresentativa dei cittadini. In particolare, non riesce a coinvolgere i cittadini giovani, a basso reddito, meno istruiti e disoccupati. Inoltre, i risultati evidenziano come mediamente il BP milanese abbia coinvolto principalmente quei cittadini che sono già attivi in politica, almeno dal punto di vista elettorale, mentre non è riuscito a coinvolgere i cittadini meno attivi. La descrizione del "partecipante medio" al BP di Milano che emerge dalla nostra indagine restituisce un cittadino anziano (50 anni o più), con un lavoro stabile e un livello di reddito in linea con la media della città (reddito tra 15.000-55.000 euro all'anno), ma con un capitale sociale (livello di istruzione terziaria e post-terziaria al 60,2 per cento contro la media milanese del 26,2 per cento e la media nazionale del 15 per cento) e con un tasso di partecipazione elettorale $(94,7$ per cento contro 
quella milanese del 63,8 per cento e quella nazionale del 67,3 per cento) e la partecipazione ad eventi politici e associazioni di volontariato cinque volte superiore alla media milanese e italiana. Si tratta di un profilo di soggetti quasi predestinati a beneficiare delle ulteriori opportunità offerte dalla democrazia partecipativa, dato l'elevato capitale sociale e associativo e la costanza della pratica della partecipazione politica nei suoi formati tradizionali. Assumendo la posizione teorica di Baiocchi e Ganuza (2014), sulla dimensione della comunicazione di BP che prevale sull'empowerment dei cittadini, e secondo i nostri risultati, i cittadini che partecipano al BP sono, in pratica, un'élite economica, culturale e professionale, che già esprime un'ampia e profonda attività di partecipazione alla vita politica e associativa della città. In questo senso, la partecipazione al BP completa un'esperienza partecipativa già significativa per questa élite culturale e partecipativa urbana.

Se l'obiettivo del BP di Milano era quello di includere soggetti esterni alla partecipazione politica e sociale (voto, adesione a partiti, sindacati, associazioni) nei nuovi modelli di attività partecipativa e dare stimolo alla non-partecipazione convenzionale al processo decisionale urbano, i risultati che emergono da questo studio denotano un fallimento. Se gli obiettivi del BP di Milano sono invece il rafforzamento, con uno strumento innovativo di partecipazione al processo decisionale urbano, del trend partecipativo delle élite sociali, culturali ed economiche della città, il processo partecipativo avviato con l'esperienza del BP ha registrato un successo. In ogni caso, avere un ritratto socio-demografico e politico completo dei partecipanti alla BP di Milano, fornisce una migliore conoscenza dei dati e delle tendenze nelle auto-selezioni dei partecipanti stessi.

Questi risultati sollecitano una riconsiderazione del metodo del bilancio partecipativo e, di conseguenza, dei metodi di democrazia partecipativa e deliberativa più in generale. Infatti, se da una prospettiva teorica queste nuove forme democratiche sono state individuate come capaci di risolvere alcuni dei problemi che affliggono le democrazie rappresentative contemporanee 
(sfiducia, astensione elettorale, frammentazione sociale e mancanza di partecipazione alla vita associativa), l'analisi della pratica dell'esperienza del BP di Milano rivela che questi metodi sono ancora lontani dall'essere in grado di assolvere al compito di responsabilizzare tutti i cittadini a nuove forme di partecipazione urbana; allo stesso tempo l'esperienza del BP milanese riesce a garantire speciali canali di comunicazione con le istituzioni urbane per quei cittadini ad alto livello di capitale sociale e culturale e con un alto tasso di partecipazione politica e sociale. Le democrazie rappresentative sono spesso accusate della loro incapacità di rispondere tempestivamente ai bisogni dei cittadini. In questo senso, includendo tutti i tipi di cittadini nel processo decisionale della città, la negoziazione delle decisioni tra i diversi gruppi rappresentativi delle parti interessate è un mezzo potente per evitare il potere illimitato dei gruppi di interesse e la loro attività di lobbying nella costruzione dell'agenda politica delle città nel contesto dei regimi urbani (Stone 1993).

$\mathrm{Al}$ contrario, se le pratiche deliberative e partecipative non riescono ad espandere la loro base partecipativa, c'è il rischio concreto che tali strumenti possano diventare strumenti ulteriori nelle mani di gruppi limitati.

Per essere in grado di rispondere alle aspettative dei cittadini e per adempiere al ruolo identificato dalla teoria, quindi, le pratiche democratiche partecipative e deliberative devono essere ulteriormente sviluppate per essere in grado di coinvolgere fasce più ampie e diversificate della popolazione. Per questo motivo, un ulteriore lavoro, sia teorico che empirico, dovrebbe focalizzare l'attenzione su come raggiungere tale scopo, con l'obiettivo ulteriore di supportare le istituzioni urbane e locali verso una più inclusiva implementazione di pratiche partecipative sull'intera comunità cittadina. Solo attraverso un'efficace integrazione tra la dimensione teorica, la ricerca sul campo e la collaborazione con le istituzioni urbane e locali coinvolte nella democrazia partecipativa, è possibile raggiungere risultati concreti e tangibili, volti ad ampliare le opportunità di partecipazione per tutti e fare del BP un modello di empowerment civico e non 
solo un canale di comunicazione più attivo tra istituzioni e cittadini auto-selezionati. 


\section{Ringraziamenti}

Ringraziamo il personale del Comune di Milano per il supporto che ha dato alla nostra ricerca. In particolare, desideriamo ringraziare Lorenzo Lipparini (Assessore alla partecipazione, cittadinanza attiva e Open Data), e Simona Bonfante (Policy Advisor alla partecipazione, cittadinanza attiva e Open Data) e tutto il loro staff, per averci permesso di somministrare il nostro questionario ai partecipanti al BP e per la revisione del questionario stesso. Inoltre, desideriamo ringraziare il Dr. Paolo Spada, ricercatore senior del progetto Empatia e tutto il suo staff, per averci concesso l'accesso ai dati BP. Infine, desideriamo ringraziare il professore Daniele Archibugi per il suo prezioso feedback su una prima bozza dell'articolo. 


\section{Bibliografia}

Abers, Rebecca, King, Robin, Votto, Daniely, and Igor Brandão. 2018. "Porto Alegre:

Participatory Budgeting and the Challenge of Sustaining Transformative Change.” World Resources Report. https://www.wri.org/wri-citiesforall/publication/porto-alegreparticipatory-budgeting-and-challenge-sustaining.

Avritzer, Leonardo. 2017. The Two Sides of Institutional Innovation: Promises and Limits of Democratic Participation in Latin America. Cheltenham, UK: Edward Elgar Publishing. Baiocchi, Gianpaolo, and Ernesto Ganuza. 2014. "Participatory Budgeting as if Emancipation Mattered.” Politics \& Society 42 (1): 29-50. https://doi.org/10.1177/0032329213512978.

Baiocchi, Gianpaolo, and Ernesto Ganuza. 2016. Popular democracy: The paradox of participation. Palo Alto, CA: Stanford University Press.

Barge, J. Kevin. 2002. "Enlarging the Meaning of Group Deliberation: From Discussion to Dialogue.” In New directions in group communication, edited by Lawrence R. Frey, 159-177. Thousand Oaks, CA: Sage Publications.

Behl, Abhishek, Sheorey, Pratima, Nayak, Sushma and Ajith V. V. Kumar. 2017. "Role of Information and Communications Technology (ICT) in Participatory Democracy: A Forerunner to an Egalitarian Society." Proceedings of the 10th International Conference on Theory and Practice of Electronic Governance, 107-116. https://doi.org/10.1145/3047273.3047375.

Cabannes, Yves. 2004. "Participatory Budgeting: A Significant Contribution to Participatory Democracy." Environment and urbanization 16 (1): 27-46. https://doi.org/10.1177/095624780401600104.

Cardoso Sampaio, Rafael. 2016. “e-Participatory Budgeting as an Initiative of e-Requests: Prospecting for Leading Cases and Reflections on e-Participation.” Revista Brasileira de Administração Pública 50 (6): 935-958. http://dx.doi.org/10.1590/0034-7612152210. 
Cellini, Marco and. Cristina Antonucci. 2020. "Report "MIPAR”. La Partecipazione dei Cittadini Milanesi al Bilancio Partecipativo attraverso la Piattaforma MilanoPartecipa." IRPPS Working papers 118/2020. https://www.movetothecloud.it/irpps/epub/index.php/wp/article/view/118.

Cini, Lorenzo. 2011. "Between Participation and Deliberation: Toward a New Standard for Assessing Democracy." 9th Pavia Graduate Conference in Political Philosophy, 4-6. https://is.muni.cz/el/1423/jaro2018/ENS296/um/teorie_literatura_hrubes/02_Cini_Betw $\underline{\text { een_Participation_and_Deliberation.pdf }}$

Dazzi, Zita. 2019. "Milano partecipa: tre giorni di incontri e dibattiti sulla cittadinanza attiva e digitale" La Repubblica, November 13. https://milano.repubblica.it/cronaca/2019/11/13/news/milano_partecipa_cittadinanza_att iva_digitale-241022945/.

De Sousa Santos, Boaventura. 1998. "Participatory Budgeting in Porto Alegre: Toward a Redistributive Democracy." Politics \& society 26 (4): 461-510. https://doi.org/10.1177/0032329298026004003.

Effing, Robin, and Bert P. Groot. 2016. "Social Smart City: Introducing Digital and Social Strategies for Participatory Governance in Smart Cities.” In Electronic Government. EGOV 2016. Lecture Notes edited by Scholl Hans et al., 241-252. Cham, CH: Springer.

European Social Survey. 2018. "Round 9 2018” (accessed May 10, 2020).

https://www.europeansocialsurvey.org/data/.

Giannattasio, Maurizio. 2016. "Nelle zone diventano realtà i progetti scelti dai cittadini”" Corriere della Sera, May 30. https://milano.repubblica.it/cronaca/2019/11/13/news/milano_partecipa_cittadinanza_att iva_digitale-241022945/. 
Hajdarowicz, Inga. 2018. "Does Participation Empower? The Example of Women Involved in Participatory Budgeting in Medellin." Journal of Urban Affairs 1-16. https://doi.org/10.1080/07352166.2018.1431048.

He, Baogang. 2011. "Civic Engagement Through Participatory Budgeting in China: Three Different Logics at Work." Public Administration and Development 31 (2): 122-133. https://doi.org/10.1002/pad.598.

Hooghe, Marc, and Sofie Marien. 2013. "A Comparative Analysis of the Relation Between Political Trust and Forms of Political Participation in Europe. European Societies 15 (1): 131-152. https://doi.org/10.1080/14616696.2012.692807.

Jabola-Carolus, Isaac, Elliott-Negri, Luke, Jasper, M. James, Mahlbacher, Jessica, Weisskircher, Manès and Anna Zhelnina. 2020. "Strategic Interaction Sequences: The Institutionalization of Participatory Budgeting in New York City.” Social Movement Studies, 19 (5-6): 640-656. https://doi.org/10.1080/14742837.2018.1505488.

Kamal, Muhammad, Sivarajah, Uthayasankar, Allegretti, Giovanni, Secchi, Michelangelo and Sofia Antunes. 2016. "Enabling Multichannel Participation Through ICT Adaptations for Participatory Budgeting." E-government 6. Available at: https://aisel.aisnet.org/amcis2016/eGov/Presentations/6/.

Kersting, Norbert., Gasparikova, Jana, Iglesias, Angel and Jelizaveta Krenjova. 2016. "Local Democratic Renewal by Deliberative Participatory Instruments: Participatory Budgeting in Comparative Study.” In Local public sector reforms in times of crisis, edited by Kuhlmann, Sabine, and Geert Bouckaert, 317-331. London: Palgrave Macmillan.

Lerner, Josh. 2014. Everyone Counts: Could "Participatory Budgeting" Change Democracy? Ithaca, NY: Cornell University Press.

Lerner, Josh. 2017. “Conclusion: Time for Participatory Budgeting to Grow Up.” New Political Science, 39(1): 156-160. https://doi.org/10.1080/07393148.2017.1278860. 
Mansbridge, Jane. 1999. “On the Idea that Participation makes Better Citizens.” In Citizen Competence and Democratic Institutions, edited by Elkin, Stephen L., and Karol Edward Soltan, 291-325. University Park, PA: Pennsylvania State University Press. Ministero dell'Interno. 2020. “Archivio storico delle elezioni” Dipartimento per gli affari interni e territoriali (Accessed May 15, 2019). https://elezionistorico.interno.gov.it/. Municipality of Milan. (2016). "Patto di Partecipazione”. June 16. https://bilanciopartecipativo.comune.milano.it/content/view/3.

Novy, Andreas, and Bernhard Leubolt. 2005. "Participatory Budgeting in Porto Alegre: Social Innovation and the Dialectical Relationship of State and Civil Society." Urban studies, 42 (11): 2023-2036. https://doi.org/10.1080/00420980500279828.

Parra, Cristhian, Rohaut, Christelle, Maeckelbergh, Marianne, Issarny, Valerie and Lames Holston. 2017. "Expanding the Design Space of ICT for Participatory Budgeting." In Proceedings of the 8th International Conference on Communities and Technologies, edited by Shuler Douglas and Myriam Lewkowicz, 213-221. New York, NY: Association for Computing Machinery.

Peña-López, Ismael. 2017. "Citizen Participation and the Rise of the Open-Source City in Spain." IT for Change. https://opendocs.ids.ac.uk/opendocs/handle/20.500.12413/13006.

Russon Gilman, Hollie, and Brian Wampler. 2019. “The Difference in Design: Participatory Budgeting in Brazil and the United States.” Journal of Public Deliberation, 5 (1): 1-7. http://doi.org/10.16997/jdd.318.

Schugurensky, Daniel. 2009. “Citizenship Participation and Participatory Democracy: Limits and Possibilities." Learning democracy by doing: Alternative practices in citizenship education and participatory democracy, 50-63. 
http://www.academia.edu/download/30849008/LDD_Proceedings_Master_Document_26_Oct_09.pdf\#page $=63$

Sintomer, Yves, Carsten Herzberg, and Anja Röcke. 2008. "Participatory Budgeting in Europe: Potentials and Challenges." International Journal of Urban and Regional Research, 32 (1): 164-178. https://doi.org/10.1111/j.1468-2427.2008.00777.x.

Sintomer, Yves, Carsten Herzberg, Röcke, Anja and Giovanni Allegretti. 2012.

"Transnational Models of Citizen Participation: The Case of Participatory Budgeting." Journal of Public Deliberation, 8 (2) Article 9. http://doi.org/10.16997/jdd.141.

Sintomer, Yves, Carsten Herzberg., Allegretti, Giovanni, Röcke, Anja and Alves, Mariana. 2013. “Participatory Budgeting Worldwide." Dialog Global, (25): 1-93. https://estudogeral.uc.pt/bitstream/10316/42267/1/Participatory\%20Budgeting\%20Worl dwide.pdf.

Sintomer, Yves, Anja Röcke, and Carsten Herzberg. 2016. Participatory Budgeting in Europe: Democracy and Public Governance. London: Routledge.

Stone, Clarence N. 1993. "Urban Regimes and the Capacity to Govern: A Political Economy Approach.” Journal of urban affairs, 15 (1), 1-28. https://doi.org/10.1111/j.14679906.1993.tb00300.x.

Talpin, Julien. 2012. Schools of Democracy: How Ordinary Citizens (sometimes) Become Competent in Participatory Budgeting Institutions. Colchester, UK: ECPR Press. Tormey, Simon. 2014. "The Contemporary Crisis of Representative Democracy." Democratic Theory, 1 (2): 104-112. https://doi.org/10.3167/dt.2014.010211.

Tormey, Simon. 2020. "Politicising Digital Space: Theory, the Internet and Renewing Democracy." Contemporary Political Theory, 19: 59-62. https://doi.org/10.1057/s41296-018-0273-9. 
Uddin, Shahzad, Yuji Mori, and Pawan Adhikari. 2019. "Participatory Budgeting in a Local Government in a Vertical Society: A Japanese Story." International Review of Administrative Sciences, 85 (3): 490-505. https://doi.org/10.1177/0020852317721335. Van der Meer, Tom W. G. 2017. "Political Trust and the 'Crisis of Democracy'." In Oxford Research Encyclopedia of Politics, edited by Jeffrey Kaplan, pp. 23. Oxford, UK: Oxford University Press.

Wampler, Brian. 2010. Participatory Budgeting in Brazil: Contestation, Cooperation, and Accountability. University Park, PA: Penn State Press.

Wampler, Brian. 2012. "Participatory Budgeting: Core Principles and Key Impacts.” Journal of Public Deliberation, 12. http://doi.org/10.16997/jdd.138.

World Value Survey. 2010. Wave 5: 2005-2009 (accessed May 10, 2020). http://www.worldvaluessurvey.org/WVSContents.jsp.

\section{Appendix}

Tabella 1. Dettaglio dei dati sui partecipanti BP, residenti a Milano e cittadini italiani.

\begin{tabular}{lccc}
\hline Età & BP & Milano & Italia \\
\hline $16-18$ & 0.29 & 2.53 & 2.86 \\
$19-29$ & 3.37 & 11.07 & 11.21 \\
$30-39$ & 11.57 & 13.74 & 11.54 \\
$40-49$ & 20.2 & 15.57 & 15.01 \\
$50-59$ & 24.16 & 15.23 & 15.77 \\
$60-70$ & 25.77 & 11.39 & 13.49 \\
$>70$ & 14.64 & 16.96 & 16.18 \\
& & & \\
\hline Reddito & & & Italia \\
\hline $0-15.000$ & BP & Milano & 44.5
\end{tabular}




$\begin{array}{lccc}15.001-55.000 & 62.53 & 52.08 & 50.04 \\ 55.001-75.000 & 16.58 & 5.11 & 3.22 \\ >75.000 & 15.42 & 7.56 & 2.24\end{array}$

\begin{tabular}{lccc}
\hline Istruzione & BP & Milano & Italia \\
\hline Nessuno/Elementare & 0.15 & 8.73 & 16.2 \\
Secondaria inferiore & 2.49 & 28.28 & 32.41 \\
Secondaria superiore & 37.19 & 36.8 & 36.43 \\
Terziaria e post-terziaria & 60.17 & 26.19 & 14.96
\end{tabular}

\begin{tabular}{lccc}
\hline Occupazione & BP & Milano & Italia \\
\hline Disoccupato & 3.42 & 3.95 & 11.45 \\
Occupato & 63.6 & 53.42 & 45.04 \\
Casalinga & 1.63 & 7.93 & 11.39 \\
Pensionato & 29.42 & 28.07 & 24.81 \\
Studente & 1.93 & 6.63 & 7.31
\end{tabular}

\begin{tabular}{lccc}
\hline Affluenza elettorale & BP & Milano & Italia \\
\hline Locale, nationale, Europee & 94.69 & 63.75 & 67.35
\end{tabular}

\begin{tabular}{lccc}
\hline Socio-political participation & BP & Milano & Italia \\
\hline Membro di un partito & 6.73 & N/A & 10.30 \\
Partecipazione a manifestazioni & 39.39 & N/A & 8.20 \\
Attività di volontariato & 47.14 & N/A & 9.20 \\
\hline
\end{tabular}

